\title{
CodRED: A Cross-Document Relation Extraction Dataset for Acquiring Knowledge in the Wild
}

\author{
Yuan Yao ${ }^{1 *}$, Jiaju Du ${ }^{1 *}$, Yankai Lin ${ }^{2}$, Peng Li ${ }^{2}$, Zhiyuan Liu ${ }^{1 \dagger}$, Jie Zhou ${ }^{2}$, Maosong Sun ${ }^{1}$ \\ ${ }^{1}$ Department of Computer Science and Technology \\ Institute for Artificial Intelligence, Tsinghua University, Beijing, China \\ Beijing National Research Center for Information Science and Technology, China \\ ${ }^{2}$ Pattern Recognition Center, WeChat AI, Tencent Inc. \\ yuan-yao18@mails.tsinghua.edu.cn, i@dujiaju.me
}

\begin{abstract}
Existing relation extraction (RE) methods typically focus on extracting relational facts between entity pairs within single sentences or documents. However, a large quantity of relational facts in knowledge bases can only be inferred across documents in practice. In this work, we present the problem of crossdocument RE, making an initial step towards knowledge acquisition in the wild. To facilitate the research, we construct the first human-annotated cross-document RE dataset CodRED. Compared to existing RE datasets, CodRED presents two key challenges: Given two entities, (1) it requires finding the relevant documents that can provide clues for identifying their relations; (2) it requires reasoning over multiple documents to extract the relational facts. We conduct comprehensive experiments to show that CodRED is challenging to existing RE methods including strong BERT-based models. We make CodRED and the code for our baselines publicly available at https://github.com/thunlp/CodRED.
\end{abstract}

\section{Introduction}

Relation extraction (RE), which aims to extract relations between entities from plain text, serves as an essential resource in populating knowledge bases (KBs) from large-scale corpora automatically. Existing RE systems typically focus on either sentence-level RE (Socher et al., 2012; Zeng et al., 2014, 2015; Lin et al., 2016; Qin et al., 2018) or document-level RE (Li et al., 2016; Peng et al., 2017; Quirk and Poon, 2017; Yao et al., 2019), and have achieved promising results on several public benchmarks. However, these works can only extract relational facts from single sentences or documents containing both two target entities, which inevitably limits the coverage of knowledge acquisition. According to our statistics on Wikipedia

\footnotetext{
* indicates equal contribution

$\dagger$ Corresponding author: Z.Liu (liuzy@tsinghua.edu.cn)
}

documents, for over $57.6 \%$ of the relational facts in Wikidata (Erxleben et al., 2014; Vrandečić and Krötzsch, 2014), the head and tail entities do not co-occur in a single document. This inspires that it is crucial to break through the limitations of document boundaries to acquire knowledge in the wild.

In this work, we make an initial step in this direction, presenting the problem of cross-document $R E$ (cross-doc RE), which requires a RE system to infer the relation between two entities by retrieving and reasoning over multiple documents. Compared to conventional sentence/document-level RE, cross-doc RE presents new challenges in two levels of granularity: (1) at the coarse-grained level, given an entity pair, RE systems are required to find multiple informative documents for each entity, instead of restricted to the sentence/document containing both entities; (2) on the fine-grained level, RE systems are required to perform both intra- and cross-document reasoning in multiple documents and then predict the relations by aggregating information. The challenges come from not only the non-trivial nature of each phase, but also the intrinsic inter-dependence among the phases.

Fig. 1 shows an example for cross-doc RE, in which Amun-her-khepeshef and Merneptah do not co-appear in a single document. To identify their relation, we need to first retrieve the relevant documents for each entity and then recognize two reasoning text paths in these documents. The first reasoning text path (the documents titled "Nefertari" and "Memeptah") shows that both target entities are the son of Ramesses II, and the second one indicates that they also share a common sister Meritamen. The information of these two reasoning text paths is complementary to each other and suggests the relation between Amun-her-khepeshef and Merneptah is sibling.

Although several datasets have been proposed for investigating cross-document reasoning (Over and Yen, 2004; Yang et al., 2018), there is still 


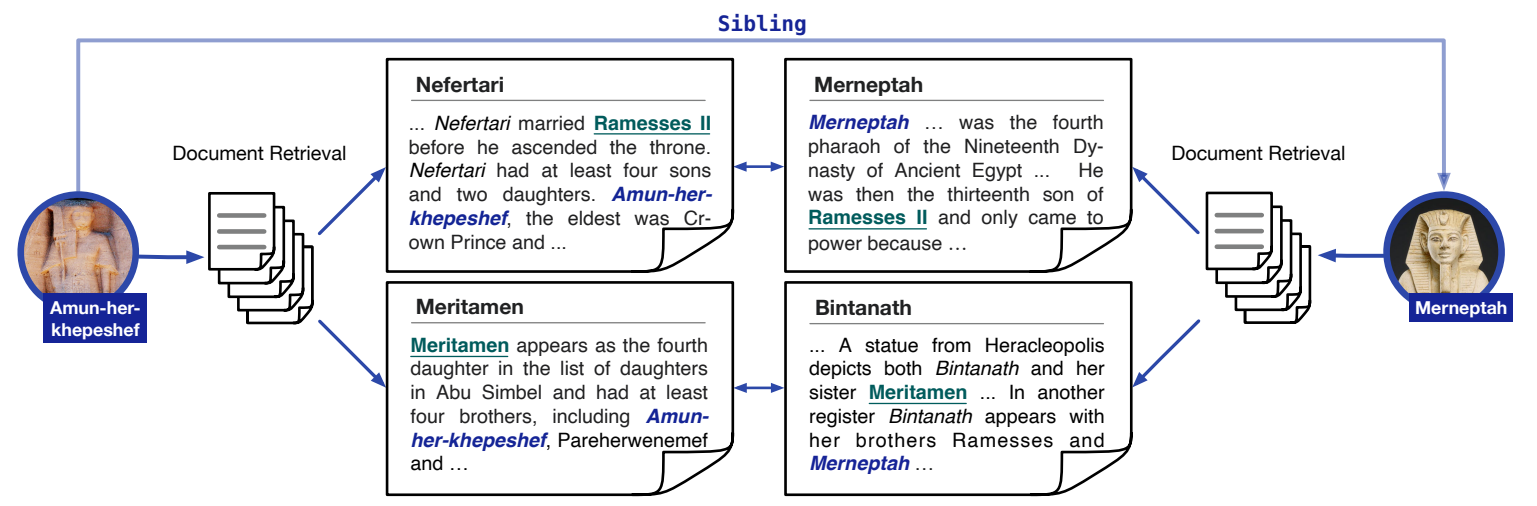

Figure 1: An example from CodRED. Two of the text paths between the target entity pair are shown. Each text path consists of two documents, which are connected by bridging entities. We only show evidence sentences in text paths for brevity. The target entity pair, bridging entity within and across documents are highlighted accordingly.

no dataset designed for cross-doc RE. To facilitate the research, we construct the first humanannotated Cross-document Relation Extraction Dataset named as CodRED, aiming to test the RE systems' ability of knowledge acquisition in the wild. CodRED has the following features: (1) it requires natural language understanding in different granularity, including coarse-grained document retrieval, as well as fine-grained cross-document multi-hop reasoning; (2) it contains 30,504 relational facts associated with 210,812 reasoning text paths, as well as enjoys a broad range of balanced relations, and long documents in diverse topics; (3) it provides strong supervision about the reasoning text paths for predicting the relation, to help guide RE systems to perform meaningful and interpretable reasoning; (4) it contains adversariallycreated hard NA instances to avoid RE models to predict relations by inferring from entity names instead of text information (Peng et al., 2020).

To assess CodRED, we propose two representative solutions based on the strong BERT-based RE architecture, including (1) a pipeline model that first extracts a relational graph for each document, and then reasons over these graphs to extract the relation; and (2) an end-to-end model that jointly considers text across different documents in text paths to predict the relation. We conduct comprehensive experiments under both closed and open settings on CodRED. Experimental results show that CodRED is very challenging to the strong BERT-based solutions, indicating ample room for further research.

\section{Data Collection}

In this section, we introduce the data collection process of the cross-doc RE dataset. Given an entity pair $(h, t)$, cross-doc RE consists of two stages: (1) Document Retrieval, which finds multiple relevant documents of given entity pairs $h$ and $t$ from a large-scale corpus $D$, which could provide clues for identifying their relationship; (2) CrossDocument Reasoning, which reasons over the retrieved documents to predict the relation.

To focus on the problem of cross-doc RE, we only annotate the relational facts where the composing entities do not co-occur in a single document. As illustrated in Fig. 1, a relational fact can be better inferred through multiple complementary reasoning text paths (i.e., two documents that contain the head and tail entity respectively, and are connected by bridging entities) in the wild. Hence, we want to construct a cross-doc RE dataset in which each instance contains a relational fact with multiple reasoning text paths as well as strong supervision about supporting evidence.

However, it is infeasible for human annotators to label multiple reasoning text paths over documents for a relational fact from scratch. We thus carefully design a principled data collection pipeline for cross-doc RE. Specifically, we construct CodRED from the English Wikipedia and Wikidata through three stages: (1) Generating distantly supervised annotations from Wikipedia documents, which serves as relation recommendations for further human annotations; (2) Annotating relations and the corresponding supporting evidence by multiple independent crowdworkers; (3) Generating adversarial hard NA instances (i.e., entity pairs and text paths that do not express positive relations) to alleviate the reasoning shortcuts in RE. Here we introduce main procedure of data collection, and we refer readers to the appendix for more details. 


\subsection{Distantly Supervised Annotation Generation}

To select relational facts and their relevant reasoning text paths for human annotations, we align Wikipedia articles with Wikidata under the distant supervision assumption (Mintz et al., 2009). To ensure the quality and encourage the diversity of the corpus, we select the articles in the Wikipedia popular page list ${ }^{1}$ as the document candidates, which cover various topics in open domain. Specially, we first recognize named entity mentions in the documents by a BERT-based (Devlin et al., 2019) named entity recognition system. Then we link these named entity mentions to Wikidata, and merge the entity mentions with same IDs in Wikidata. Finally, we align each named entity pair from two different documents with their relations in Wikidata to form one reasoning text path of this entity pair.

However, different from previous works that adopt sentence-level (Riedel et al., 2010; Han et al., 2018) or document-level (Yao et al., 2019) distant supervision, we find that directly performing distant supervision for entities across documents will lead to substantial noise (i.e., over $95 \%$ raw relation labels from distant supervision are not expressed in the given text paths, according to manual verification on distantly supervised samples). To address the problem, we introduce additional requirements that there exists at least one relational reasoning chain between the target entity pair in two documents. Here, the reasoning chain is defined as a relational path between the entity pair $(h, t)$, which is bridged by another entity $e$ appearing in both documents, such that $e$ has relation with $h$ and $t$ in Wikidata respectively. The reasoning chain can be formally denoted as $h \stackrel{r_{i}}{\longrightarrow} e \stackrel{r_{j}}{\longrightarrow} t^{2}$. For example, in Fig. 1, Amun-her-khepeshef and Merneptah are linked by a reasoning chain consisting of two relational facts: Amun-her-khepeshef $\stackrel{\text { father }}{\longrightarrow}$ Ramesses II $\stackrel{\text { child }}{\longrightarrow}$ Merneptah. To alleviate the noise in reasoning chains, we ask experts to manually filter out frequent reasoning chains that cannot induce the target relations. We observe that this constraint can substantially alleviate the wrong-labeling problem, with less than $45 \%$ noise in the improved distantly supervised annotations.

In addition, we further sub-sample the annota-

\footnotetext{
${ }^{1}$ https://en.wikipedia.org/wiki/ Wikipedia:Lists_of_popular_pages_by_ WikiProject

${ }^{2} r_{i}$ and $r_{j}$ can be relations or inverse relations in Wikidata.
}

tions of frequent relations for two reasons: (1) to balance the relation distribution; (2) to prevent the strong correlation between the relations and documents (i.e., we make sure that the co-occurrence of any relation and document is fewer than 20), inspired by Welbl et al. (2018).

\subsection{Human Annotation Generation}

After obtaining distantly supervised relation annotations, we ask human annotators to label them to remove the noisy annotations in distant supervision. To ensure the dataset quality, we provide principled guidelines as well as training to the annotators, and utilize a test task to examine if the annotators understand our annotation principle. We also conduct regular quality inspections for each annotator, and update the feedback in the individual reports.

During the annotation, human annotators are asked to label (1) text paths, i.e., whether a relational fact can be supported by the given text path without external knowledge, and (2) evidence sentences, i.e., selecting a set of evidence sentences (if any) from the reasoning text path that can fully support the relational fact. Each reasoning text path is annotated independently by at least two annotators, and will be further annotated by a third annotator if there are disagreements in whether the relational fact can be supported.

After human annotation, each entity pair is associated with multiple reasoning text paths, which are labeled with either positive relations, or NA indicating no relation. The final relations between an entity pair are aggregated from all paths in between, by the union of the positive relations in each path. The final relation will be NA if there is no positive path in between. We discard the text paths if the relations can be extracted from one document, i.e., there are evidence annotations in only one document.

\subsection{Adversarial NA Instance Generation}

We find obvious reasoning shortcuts in our and most existing RE datasets (Peng et al., 2020), i.e., there are obvious correlations between some relations and entity names. This makes RE models could easily infer the relations from the entity names without performing complex reasoning in text, which may over-estimate their performance. To overcome this problem, we employ a novel adversarial NA instance generation strategy at entitylevel, which requires RE models to pay more attention to understanding text. Moreover, we also add 


\begin{tabular}{l|rr|rr}
\hline \multirow{2}{*}{ Set } & \multicolumn{2}{|c|}{ \#Fact } & \multicolumn{2}{c}{ \#Path } \\
& Pos. & \multicolumn{1}{c}{ NA } & Pos. & \multicolumn{1}{c}{ NA } \\
\hline Train & 2,733 & 16,668 & 8,623 & 120,925 \\
Dev & 1,010 & 4,558 & 2,558 & 38,182 \\
Test & 1,012 & 4,523 & 2,505 & 38,019 \\
\hline
\end{tabular}

Table 1: Statistics of data split. (\#Fact: the number of relational facts; \#Path: the number of reasoning text paths; Pos.: Positive.)

challenging path-level NA instances to test RE models' ability in reasoning in the presence of noise (i.e., there are NA text paths between entity pairs), which is important in real-world applications.

Entity-Level Adversarial NA Instance. We select challenging adversarial NA entity pairs, i.e., entity pairs that do not have relations in Wikidata but are assigned with high confidence of positive relations by RE models. Specifically, we first train a series of RE classifiers (i.e., CNN, LSTM and BERT, etc.) that extract the relations based on entity names. ${ }^{3}$ Then for each positive entity pair $(h, t)$, we generate an NA entity pair by replacing $h$ or $t$ with the top entity ranked by the confidence of the ensemble models. We generate 23, 069 adversarial NA entity pairs in total, reducing the percentage of positive instances to $15.6 \%$ in the dataset. ${ }^{4}$

Path-Level Adversarial NA Instance. To test the model ability of cross-document reasoning in the presence of noise in closed setting (see Sec. 3), we generate NA reasoning text paths for both humanannotated and adversarial NA entity pairs. Given an entity pair, we enumerate all possible reasoning text paths consisting of two documents that contain head and tail entities respectively, and share at least one common entity. To select hard NA paths, we choose the reasoning text paths that have the most shared entities between the composing documents.

\section{Post-Processing and Benchmarks}

We first introduce the data split process, including the split of positive and NA entity pairs. (1) Positive entity pair split. We split the positive entity pairs into training, development and test set, such that there is no overlap in entity names under the same positive relations, to prevent the correlation between relations and entity names. (2) NA entity pair split. Adversarially-created NA entity pairs

\footnotetext{
${ }^{3}$ Here we use entity names to predict the relations, since we find it can effectively eliminate the reasoning shortcuts in our experiments, and also has better computation efficiency.

${ }^{4}$ The percentage reflects the sparsity of positive relations in real-world RE scenarios (Zhang et al., 2017).
}

(see Sec. 2.3) are randomly split into the three sets. Human-annotated NA entity pairs (see Sec. 2.2) are only put into training set to avoid the situation that there are unlabeled positive paths between the entity pair in open domain corpora, which could lead to false negative in evaluation in open setting (see following sections). Table 1 shows the statistics.

Since CodRED requires natural language understanding in different granularity, we design two benchmark settings to fully evaluate each required capability including (1) document retrieval, and (2) cross-document reasoning.

Closed Setting. In this setting, we test model capabilities in cross-document reasoning. Given an entity pair, RE models need to extract relations based on the given positive text paths and NA text paths. The first challenge comes from intra- and cross-document multi-hop reasoning in each text path. RE models need to first resolve complex interactions between entities within long documents, which may require logical, coreference and commonsense reasoning (Yao et al., 2019). Then RE models have to overcome the semantic gap between documents, and perform cross-document multi-hop reasoning through multiple potential bridging entities ( 4.7 on average) to establish the relation in each reasoning text path. The second challenge is that RE models need to synthesize all information in multiple text paths to obtain the final relation.

Open Setting. This setting fully tests the ability of RE in the wild. Given a target entity pair, models need to first retrieve relevant documents for the entity pair from full English Wikipedia corpus $(5,882,234$ documents in total, 3,646 reasoning text path candidates for each entity pair on average), then perform cross-document reasoning with the retrieved documents to predict the relation. Compared with natural language queries in open domain question answering (Chen et al., 2017), the sparse query information in entity pairs presents unique challenges to document retrieval ability. The second challenge comes from both the quadratic number of potential paths (efficiency), and the finegrained influence of document retrieval on the extraction of relations (effectiveness).

\section{Data Analysis}

In this section, we present data analysis of CodRED, including data statistics, required abilities in our dataset, and cross-document relation instances.

Data Statistics. CodRED enjoys diversity in open 


\begin{tabular}{lcccc}
\hline Dataset & DR & CDR & IDR & ISR \\
\hline TACRED & & & & $\checkmark$ \\
FewRel & & & & $\checkmark$ \\
KnowledgeNet & & & & $\checkmark$ \\
BC5CDR & & & $\checkmark$ & $\checkmark$ \\
DialogRE & & & $\checkmark$ & $\checkmark$ \\
DocRED & & & $\checkmark$ & $\checkmark$ \\
CodRED & $\checkmark$ & $\checkmark$ & $\checkmark$ & $\checkmark$ \\
\hline
\end{tabular}

Table 2: Abilities required in different RE datasets. (DR: document retrieval, CDR: cross-document reasoning, IDR: intra-document reasoning, ISR: intrasentence reasoning.)

domain in two aspects: relations and documents. (1) Relations. CodRED covers 276 relation types in different domains, including science $(24.6 \%)$, work $(21.3 \%)$ and art $(8.7 \%)$, etc. Besides, CodRED contains 4,755 positive relational facts and 13,686 positive reasoning text paths, along with 25, 749 NA relational facts and 197, 126 NA reasoning text paths. CodRED exhibits balanced relation distribution, where the most frequent positive relation accounts for less than $4.5 \%$. (2) Documents. The documents cover a variety of topics, including geography $(28.7 \%)$, entertainment $(19.6 \%)$, and society $(8.5 \%)$, etc. The average length of documents is 2, 416 words, presenting challenges for modeling long text in both efficiency and effectiveness. We refer readers to the appendix for more details.

Required Abilities. We compare required abilities of CodRED with existing RE datasets in Table 2, including (1) sentence-level RE datasets TACRED (Zhang et al., 2017), FewRel (Han et al., 2018) and KnowledgeNet (Mesquita et al., 2019), and (2) document-level RE datasets BC5CDR (Li et al., 2016), DocRED (Yao et al., 2019) and DialogRE (Yu et al., 2020). Compared with existing $\mathrm{RE}$ datasets that mainly focus on extracting relations from local contexts, i.e., single sentences or documents, CodRED presents unique challenges in document retrieval and cross-document reasoning.

Intra- and Cross-Document Reasoning. Crossdoc RE requires both intra- and cross-document multi-hop reasoning. For intra-document reasoning, we randomly sample 500 positive reasoning text paths and annotate the number of hops needed within the documents. 1.3 hops are required within documents on average, indicating that there are 2.6 hops in each path on average. For cross-document reasoning, a crucial challenge comes from multiple potential bridging entities between documents ( 4.7 on average). Each reasoning text path is labeled with 4.8 supporting sentences on average, account- ing for $2.7 \%$ sentences in each path. This means that models need to select correct and meaningful sentences and bridging entities for cross-document reasoning from rich context and severe distractions.

\section{Baselines}

In this section, we design baseline models to assess the challenge of CodRED. In the closed setting, we design two representative baselines that perform cross-document reasoning based on strong architectures, including: (1) a pipeline model that first extracts a relational graph (i.e., graph containing entities and their relations) for each document, and then reasons over these graphs to extract the relation; and (2) an end-to-end model that jointly considers text across different documents in text paths to predict the relation. In the open setting, we first retrieve relevant documents and connect them into text paths, and then perform cross-document reasoning to predict the relation. We refer readers to the appendix for implementation details.

\subsection{Document Retrieval}

In the open setting, given an entity pair $(h, t)$ and a document set $D$ (i.e., full Wikipedia corpus), we first find relevant documents to extract their relation. Due to the large number of possible documents containing $h$ and $t$ respectively, we explore several strategies to retrieve the relevant documents and connect them into text paths. Specifically, we enumerate all possible text paths between the target entity pairs (i.e., two documents that contain $h$ and $t$ respectively with shared entities) as candidates. We first present a random baseline, where the candidate paths are randomly sampled. We also experiment with several heuristic retrieval strategies, where text paths are ranked by the heuristic scores. Specifically, the score of a text path $\left(d_{h}, d_{t}\right)$ is given by: (1) entity count: multiplication of the occurrence number of $h$ in $d_{h}$ and the occurrence number of $t$ in $d_{t}$, (2) shared entity: number of shared entities that appears in both $d_{h}$ and $d_{t}$, or (3) TF-IDF: TF-IDF similarity (Manning et al., 2008) between the two documents. After ranking, we select top $K$ paths with highest scores $\left\{\left(d_{h}^{i}, d_{t}^{i}\right)\right\}_{i=0}^{K}$.

\subsection{Cross-Document Reasoning}

Given the text paths between an entity pair, we present two baselines that perform cross-document reasoning for cross-doc RE, including a pipeline model and an end-to-end model. 


\subsubsection{Pipeline Model}

We build a pipeline model that decomposes crossdocument reasoning into three phases as follows:

1. Intra-Document Relational Graph Extraction. We predict the relations between the entities within each document containing head or tail entities using a BERT-based document-level RE model, resulting in a relational graph for each document.

2. Cross-Document Relation Reasoning. For each possible bridging entity $e$ (i.e., any entity shared by two relational graphs), we predict the relation between the target entity pair based on the entity types of $h, t$ and $e$, and relation $r_{i}$ between $(h, e)$, as well as the relation $r_{j}$ between $(e, t)$. Note that the prediction is only based on the relational graphs without considering text. Specifically, we feed the concatenation of the embeddings of $r_{i}$ and $r_{j}$ and embeddings of the types of $h, t$ and $e$ (e.g., person, organization and location) into a fully connected layer to obtain the relation distribution.

3. Relation Aggregation. We finally obtain the relation between the target entity pair by aggregating relation scores from all bridging entities. For each relation, the aggregated score is obtained by the max relation score from all possible bridging entities in all text paths.

\subsubsection{End-to-end Model}

Despite their simplicity, pipeline models usually suffer from error propagation. We also design an end-to-end model that jointly considers text across documents in text paths to predict the relation.

Specifically, given a text path, we adopt BERT as the text encoder. Since intra- and cross-document text understanding are both important components in cross-doc RE, we introduce two relation prediction tasks, including: (1) Intra-document relation prediction, where the model is asked to predict intra-document relations labeled by distant supervision as in Yao et al. (2019). (2) Cross-document relation prediction, where the model needs to predict cross-document relations labeled in CodRED.

Specifically, in cross-document relation prediction, documents are first concatenated and then tokenized. Then we add entity markers to mark the positions of head/tail/bridging entity mentions. The tokens are fed into BERT to obtain the text path representation $\mathbf{p}_{i}$. After that, to select meaningful paths in the presence of noise, following previous works on distantly supervised RE, we synthesize all informative paths by selective attention mechanism (Lin et al., 2016) and obtain the aggre- gated representation $\mathrm{x}$. The aggregated entity pair representation $\mathbf{x}$ is then fed into a fully connected layer followed by a softmax layer to obtain the distribution of the relation between the entity pair.

Besides the entity-level supervision, we also incorporate path-level supervision using an auxiliary classification task, where models need to predict the relation expressed in each path based on $\mathbf{p}_{i}$.

\section{Experiments}

In this section, we assess the challenges of CodRED in both closed and open benchmark settings.

\subsection{Evaluation Metrics}

In closed setting, following previous works (Zeng et al., 2015; Lin et al., 2016), we evaluate our model using aggregate precision-recall curves, and report the area under curve (AUC), the maximum F1 on the curve and Precision@K (P@K). In open setting, we first retrieve relevant documents (top 16 paths) from full Wikipedia corpus, and then use the models trained in the closed setting to infer the relation between the entity pair. We report the mean average precision (MAP), Recall@K (R@K) and mean reciprocal rank (MRR) to show the performance of document retrieval.

\subsection{Overall Results}

We report experimental results in both settings in Table 3, where document retrieval in open setting is based on the best performing entity count strategy. From the results we observe that: (1) The overall performance in the two benchmark settings is unsatisfactory for both baseline models, demonstrating the challenge of cross-doc RE. (2) The end-to-end model consistently outperforms the pipeline model by a large margin in both settings. This indicates that the pipeline model, i.e., simple adaptation of existing document-level RE approaches, cannot well handle cross-doc RE. The results show the necessity of developing RE models that jointly model text across different documents tailored for crossdoc RE. (3) The performance of models in open setting is significantly lower than their counterparts in closed setting. Document retrieval results in Table 4 also indicate that simple heuristic retrieval strategies cannot well serve cross-doc RE. In summary, the results show that CodRED is challenging to existing RE models, where retrieving relevant documents in open domain and reasoning over multiple documents present their unique challenges. 


\begin{tabular}{|c|c|c|c|c|c|c|c|c|c|}
\hline \multirow{2}{*}{ Setting } & \multirow{2}{*}{ Model } & \multicolumn{4}{|c|}{ Dev } & \multicolumn{4}{|c|}{ Test } \\
\hline & & AUC & $\mathrm{F} 1$ & P@500 & P@ 1000 & AUC & $\mathrm{F} 1$ & P@500 & P@ 1000 \\
\hline \multirow{2}{*}{ Closed } & Pipeline & 17.45 & 30.54 & 30.60 & 26.70 & 18.94 & 32.29 & 32.00 & 28.70 \\
\hline & End-to-end & 47.94 & 51.26 & 62.80 & 51.00 & 47.46 & 51.02 & 65.00 & 51.20 \\
\hline \multirow{2}{*}{ Open } & Pipeline & 14.07 & 26.45 & 27.00 & 19.90 & 16.26 & 28.70 & 30.00 & 24.50 \\
\hline & End-to-end & 40.86 & 47.23 & 59.00 & 46.30 & 39.05 & 45.06 & 57.80 & 45.10 \\
\hline
\end{tabular}

Table 3: Main results in two benchmark settings.

\begin{tabular}{lrrrr}
\hline Strategy & MAP & MRR & R@ 16 & R@ 100 \\
\hline Random & 3.48 & 3.86 & 9.22 & 22.02 \\
Shared Entity & 7.61 & 8.25 & 19.74 & 42.14 \\
TF-IDF & 8.32 & 9.05 & 20.02 & 34.37 \\
Entity Count & $\mathbf{1 9 . 8 3}$ & $\mathbf{2 3 . 8 7}$ & $\mathbf{3 6 . 7 3}$ & $\mathbf{5 9 . 6 6}$ \\
\hline
\end{tabular}

Table 4: Document retrieval results on the dev set.

\subsection{Analysis}

To provide better understanding of cross-doc RE and CodRED, we conduct comprehensive experiments and analysis. Unless otherwise specified, all the experiments and analysis are conducted in the closed setting on the development set.

Path-level Supervision. To investigate the effect of path-level annotations (i.e., annotation indicating whether the path expresses positive or NA relations) for cross-doc RE, we remove path-level supervision and report the results in Table 5, from which we observe that: (1) The model performance degrades in the closed setting when path-level supervision is removed. It indicates path-level supervision could effectively help to filter out the noise within multiple reasoning text paths. (2) In the open setting, the advantage of models supervised by path-level annotations shrinks. We hypothesize the reason is that the retrieved text paths in the open setting exhibit different distributions from the training set, making it difficult for the models to find the positive paths.

To verify the aforementioned hypothesis, we further evaluate the performance of relation classification given golden positive text paths and evidence sentences in the closed setting. Specifically, we remove the NA entity pairs, since they do not have golden positive text paths or evidence. Given an entity pair, we compare the performance of models that during evaluation are provided with (1) all text paths in between, (2) golden positive text paths, (3) golden evidence sentences. Results in Fig. 2 show that: (1) The performance of the endto-end model improves significantly when golden positive text paths and evidence are given. This

\begin{tabular}{ll|c|ccc}
\hline Setting & Model & Sup. & AUC & F1 & P@500 \\
\hline \multirow{4}{*}{ Closed } & \multirow{2}{*}{ Pipeline } & & 16.65 & 30.54 & 26.20 \\
& & $\checkmark$ & $\mathbf{1 7 . 4 5}$ & $\mathbf{3 0 . 8 5}$ & $\mathbf{3 0 . 6 0}$ \\
\cline { 2 - 6 } & \multirow{2}{*}{ End-to-end } & & 45.30 & 48.55 & 60.80 \\
& & $\checkmark$ & $\mathbf{4 7 . 9 4}$ & $\mathbf{5 1 . 2 6}$ & $\mathbf{6 2 . 8 0}$ \\
\hline \multirow{4}{*}{ Open } & \multirow{2}{*}{ Pipeline } & & 13.52 & 26.45 & 22.40 \\
& & $\checkmark$ & $\mathbf{1 4 . 0 7}$ & $\mathbf{2 6 . 5 1}$ & $\mathbf{2 7 . 0 0}$ \\
\cline { 3 - 6 } & \multirow{2}{*}{ End-to-end } & & $\mathbf{4 1 . 8 9}$ & 46.19 & 57.40 \\
& & $\checkmark$ & 40.86 & $\mathbf{4 7 . 2 3}$ & $\mathbf{5 9 . 0 0}$ \\
\hline
\end{tabular}

Table 5: Ablation results on path-level supervision in two benchmark settings. Sup.: path-level supervision.

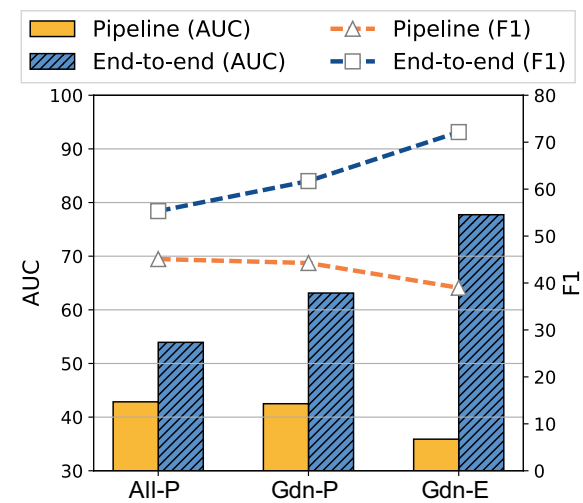

Figure 2: Experimental results when golden text paths or evidence sentences are given. All-P: all text paths, Gdn-P: golden text paths, Gdn-E: golden evidence.

shows the importance and challenge of information selection from rich context for cross-doc RE. (2) The performance of the pipeline model degrades, since the number of reasoning chains in golden paths/evidence is very limited, which leads to over-fitting. (3) Extracting relations is challenging in CodRED even if golden evidence sentences are given, since models need to perform reasoning across multiple sentences and also overcome the semantic gap between different documents. In summary, the results indicate ample room for improvement in both selecting relevant information and reasoning over complex context.

Intra- v.s. Cross-Document Supervision. To investigate the importance of intra- and crossdocument text understanding to cross-doc RE, 


\begin{tabular}{lcccc}
\hline Model & AUC & F1 & P@ 500 & P@1000 \\
\hline End-to-end & $\mathbf{4 7 . 9 4}$ & $\mathbf{5 1 . 2 6}$ & $\mathbf{6 2 . 8 0}$ & $\mathbf{5 1 . 0 0}$ \\
w/o ID-Sup & 15.67 & 26.56 & 33.20 & 26.50 \\
w/o CD-Sup & 10.14 & 17.21 & 19.00 & 15.40 \\
\hline
\end{tabular}

Table 6: Ablation study on intra-document supervision (ID-Sup) and cross-document supervision (CD-Sup).

we ablate the corresponding supervision (see Sec. 5.2.2) and report the results in Table $6 .{ }^{5} \mathrm{We}$ observe dramatic drops in performance when removing either intra- or cross-document supervision. This shows that cross-doc RE requires deep text understanding both within and across documents.

Entity Names v.s. Context. Previous works have shown that RE systems tend to exploit shallow clues in existing datasets, i.e., predict relations based on entity names, instead of inferring from contexts (Peng et al., 2020). To investigate the contribution of each information source in CodRED, we ablate each information source and report the results in Table 7: (1) Entity Only. Models are given only names of the entity pair to predict their relation. (2) Context Only. The mentions of head and tail entities in documents are replaced by special mask tokens. Experimental results show that models struggle to predict relations only from entity names, and masking entity names does not dramatically hurt the performance. This indicates that there are no obvious correlations between relations and entity names in CodRED, due to the existence of adversarial NA entity pairs (see Sec. 2.3). In summary, although entity names can provide useful information in many cases, CodRED encourages $\mathrm{RE}$ models to infer relations by reasoning in rich context, instead of relying on shallow correlation between relations and entity names. In this sense, CodRED provides a more reasonable benchmark for knowledge acquisition systems.

\section{Related Work}

A variety of RE datasets have been constructed to promote the development of RE systems in recent years, which can be categorized in two main categories: (1) Sentence-level RE datasets focus on extracting relations on sentence-level, where the composing entities of a relational fact must co-appear in single sentences, with the relations labeled by human annotators (Doddington et al., 2004; Walker et al., 2006; Hendrickx et al., 2010;

\footnotetext{
${ }^{5}$ The results do not include the pipeline model, since both supervisions are necessary for the model to infer the relation.
}

\begin{tabular}{l|cc|ccc}
\hline Model & Ent. & Ctx. & AUC & F1 & P@ 500 \\
\hline Entity Only & $\checkmark$ & & 10.46 & 21.19 & 21.70 \\
\hline \multirow{2}{*}{ Pipeline } & & $\checkmark$ & 12.72 & 25.46 & 25.40 \\
& $\checkmark$ & $\checkmark$ & 17.45 & 30.54 & 30.60 \\
\hline \multirow{2}{*}{ End-to-end } & $\checkmark$ & $\checkmark$ & 41.76 & 47.33 & 58.60 \\
& $\checkmark$ & $\checkmark$ & $\mathbf{4 7 . 9 4}$ & $\mathbf{5 1 . 2 6}$ & $\mathbf{6 2 . 8 0}$ \\
\hline
\end{tabular}

Table 7: Ablation results on entity names (Ent.) and context (Ctx.).

Han et al., 2018; Mesquita et al., 2019) or distant supervision (Riedel et al., 2010; Zhang et al., 2017; Elsahar et al., 2018). (2) Cross-sentence RE datasets focus on extracting cross-sentence relations from documents ( $\mathrm{Li}$ et al., 2016; Peng et al., 2017; Quirk and Poon, 2017; Yao et al., 2019) or dialogues (Yu et al., 2020). Notably, NIST TAC SM-KBP 2019 Track $^{6}$ aims to extract and link document-level KBs from different languages and modalities. However, these datasets are still limited at sentence-level or document-level without considering cross-document reasoning, which restricts the coverage of knowledge acquisition. Hence, we extend RE to cross-document level, and construct a large-scale human-annotated dataset CodRED to facilitate further research.

Cross-document natural language understanding has received increasing interest in recent years. Several datasets have been constructed including cross-document question answering (Yang et al., 2018; Welbl et al., 2018) and cross-document summarization (Over and Yen, 2004; Owczarzak and Dang, 2011; Fabbri et al., 2019). In comparison with existing datasets, our dataset is tailored for the task of RE with fine-grained path and evidence annotations, and investigates the more open and challenging scenario of knowledge acquisition.

\section{Conclusion}

In this work, we study the problem of cross-doc RE. To facilitate the research for the problem, we present the first human-annotated dataset CodRED, and propose two representative solutions. Experimental results show that CodRED is challenging for strong RE models, indicating ample room for improvement. In this work, we focus on acquiring knowledge from text paths consisting of two documents. In the future, we plan to further explore longer text paths to better facilitate knowledge acquisition in the wild.

\footnotetext{
${ }^{6}$ https: //tac.nist.gov/2019/SM-KBP/ index.html
} 


\section{Ethical Considerations}

In this section, we discuss the main ethical considerations of CodRED dataset: (1) Intellectual property protection. CodRED is constructed from Wikipedia and Wikidata, of which permissions are granted to copy, distribute and modify the contents under the terms of the Creative Commons AttributionShareAlike 3.0 Unported License and Creative Commons CC0 License respectively. (2) Privacy. The data collection procedure is designed for factual knowledge acquisition, and does not involve privacy issues. (3) Compensation. During relation annotation, the salary for annotating each relation instance is determined by the average time of annotation and local labor compensation standard. (4) Data characteristics. We refer readers to the appendix and data description file for more detailed characteristics of the dataset. (5) Potential problems. While principled measures are taken to ensure the quality of the dataset, there might still be potential problems with the dataset quality, which may lead to incorrect predictions in knowledge acquisition applications. However, moderate noise is common in large-scale modern KBs, even for human contributed contents, which should not cause serious issues.

\section{Acknowledgement}

This work is jointly funded by the Natural Science Foundation of China (NSFC) and the German Research Foundation (DFG) in Project Crossmodal Learning, NSFC 62061136001 / DFG TRR-169.

\section{References}

Livio Baldini Soares, Nicholas FitzGerald, Jeffrey Ling, and Tom Kwiatkowski. 2019. Matching the blanks: Distributional similarity for relation learning. In Proceedings of ACL, pages 2895-2905.

Danqi Chen, Adam Fisch, Jason Weston, and Antoine Bordes. 2017. Reading Wikipedia to answer opendomain questions. In Proceedings of $A C L$, pages 1870-1879.

Jacob Devlin, Ming-Wei Chang, Kenton Lee, and Kristina Toutanova. 2019. BERT: pre-training of deep bidirectional transformers for language understanding. In Proceedings of NAACL: HLT, pages 4171-4186.

George Doddington, Alexis Mitchell, Mark Przybocki, Lance Ramshaw, Stephanie Strassel, and Ralph Weischedel. 2004. The automatic content extraction
(ACE) program-tasks, data, and evaluation. In Proceedings of LREC, pages 837-840.

Hady Elsahar, Pavlos Vougiouklis, Arslen Remaci, Christophe Gravier, Jonathon Hare, Frederique Laforest, and Elena Simperl. 2018. T-REx: A large scale alignment of natural language with knowledge base triples. In Proceedings of LREC.

Fredo Erxleben, Michael Günther, Markus Krötzsch, Julian Mendez, and Denny Vrandecic. 2014. Introducing wikidata to the linked data web. In Proceedings of ISWC, pages 50-65.

Alexander Fabbri, Irene Li, Tianwei She, Suyi Li, and Dragomir Radev. 2019. Multi-news: A large-scale multi-document summarization dataset and abstractive hierarchical model. In Proceedings of $A C L$, pages 1074-1084.

$\mathrm{Xu}$ Han, Hao Zhu, Pengfei Yu, Ziyun Wang, Yuan Yao, Zhiyuan Liu, and Maosong Sun. 2018. FewRel: A large-scale supervised few-shot relation classification dataset with state-of-the-art evaluation. In Proceedings of EMNLP, pages 4803-4809.

Iris Hendrickx, Su Nam Kim, Zornitsa Kozareva, Preslav Nakov, Diarmuid Ó Séaghdha, Sebastian Padó, Marco Pennacchiotti, Lorenza Romano, and Stan Szpakowicz. 2010. SemEval-2010 task 8: Multi-way classification of semantic relations between pairs of nominals. In Proceedings of $A C L$, pages 33-38.

Jiao Li, Yueping Sun, Robin J. Johnson, Daniela Sciaky, Chih-Hsuan Wei, Robert Leaman, Allan Peter Davis, Carolyn J. Mattingly, Thomas C. Wiegers, and Zhiyong Lu. 2016. BioCreative V CDR task corpus: a resource for chemical disease relation extraction. Database, pages 1-10.

Yankai Lin, Shiqi Shen, Zhiyuan Liu, Huanbo Luan, and Maosong Sun. 2016. Neural relation extraction with selective attention over instances. In Proceedings of ACL, pages 2124-2133.

Christopher D Manning, Prabhakar Raghavan, and Hinrich Schütze. 2008. Introduction to information retrieval. Cambridge university press.

Filipe Mesquita, Matteo Cannaviccio, Jordan Schmidek, Paramita Mirza, and Denilson Barbosa. 2019. KnowledgeNet: A benchmark dataset for knowledge base population. In Proceedings of EMNLP-IJCNLP, pages 749-758.

Mike Mintz, Steven Bills, Rion Snow, and Daniel Jurafsky. 2009. Distant supervision for relation extraction without labeled data. In Proceedings of $A C L$, pages 1003-1011.

Paul Over and James Yen. 2004. An introduction to duc-2004. In Proceedings of DUC.

Karolina Owczarzak and Hoa Trang Dang. 2011. Overview of the tac 2011 summarization track: Guided task and aesop task. In Proceedings of TAC. 
Hao Peng, Tianyu Gao, Xu Han, Yankai Lin, Peng Li, Zhiyuan Liu, Maosong Sun, and Jie Zhou. 2020 Learning from context or names? an empirical study on neural relation extraction. In Proceedings of EMNLP, pages 3661-3672.

Nanyun Peng, Hoifung Poon, Chris Quirk, Kristina Toutanova, and Wen-tau Yih. 2017. Cross-sentence n-ary relation extraction with graph LSTMs. TACL, 5:101-115.

Pengda Qin, Weiran Xu, and William Yang Wang. 2018. Robust distant supervision relation extraction via deep reinforcement learning. In Proceedings of $A C L$, pages 2137-2147.

Chris Quirk and Hoifung Poon. 2017. Distant supervision for relation extraction beyond the sentence boundary. In Proceedings of EACL, pages 11711182.

Sebastian Riedel, Limin Yao, and Andrew McCallum. 2010. Modeling relations and their mentions without labeled text. In Proceedings of ECML-PKDD, pages 148-163.

Richard Socher, Brody Huval, Christopher D. Manning, and Andrew Y. Ng. 2012. Semantic compositionality through recursive matrix-vector spaces. In Proceedings of EMNLP-CoNLL, pages 1201-1211.

Denny Vrandečić and Markus Krötzsch. 2014. Wikidata: a free collaborative knowledgebase. Соттиnications of the ACM.

Christopher Walker, Stephanie Strassel, Julie Medero, and Kazuaki Maeda. 2006. ACE 2005 multilingual training corpus. Linguistic Data Consortium, Philadelphia, 57.

Johannes Welbl, Pontus Stenetorp, and Sebastian Riedel. 2018. Constructing datasets for multi-hop reading comprehension across documents. TACL, 6:287-302.

Thomas Wolf, Lysandre Debut, Victor Sanh, Julien Chaumond, Clement Delangue, Anthony Moi, Pierric Cistac, Tim Rault, R'emi Louf, Morgan Funtowicz, and Jamie Brew. 2019. Huggingface's transformers: State-of-the-art natural language processing.

Yonghui Wu, Mike Schuster, Zhifeng Chen, Quoc V Le, Mohammad Norouzi, Wolfgang Macherey, Maxim Krikun, Yuan Cao, Qin Gao, Klaus Macherey, et al. 2016. Google's neural machine translation system: Bridging the gap between human and machine translation. arXiv preprint arXiv:1609.08144.

Zhilin Yang, Peng Qi, Saizheng Zhang, Yoshua Bengio, William W. Cohen, Ruslan Salakhutdinov, and Christopher D. Manning. 2018. HotpotQA: A dataset for diverse, explainable multi-hop question answering. In Processing of EMNLP, pages 23692380 .
Yuan Yao, Deming Ye, Peng Li, Xu Han, Yankai Lin, Zhenghao Liu, Zhiyuan Liu, Lixin Huang, Jie Zhou, and Maosong Sun. 2019. DocRED: A large-scale document-level relation extraction dataset. In Proceedings of ACL, pages 764-777.

Dian Yu, Kai Sun, Claire Cardie, and Dong Yu. 2020. Dialogue-based relation extraction. In Proceedings of ACL, pages 4927-4940.

Daojian Zeng, Kang Liu, Yubo Chen, and Jun Zhao. 2015. Distant supervision for relation extraction via piecewise convolutional neural networks. In Proceedings of EMNLP, pages 1753-1762.

Daojian Zeng, Kang Liu, Siwei Lai, Guangyou Zhou, and Jun Zhao. 2014. Relation classification via convolutional deep neural network. In Proceedings of COLING, pages 2335-2344.

Yuhao Zhang, Victor Zhong, Danqi Chen, Gabor Angeli, and Manning Christopher D. 2017. Positionaware attention and supervised data improve slot filling. In Proceedings of EMNLP, pages 35-45. 


\section{A Data Collection Details}

Named Entity Annotation. To generate distantly supervised relation annotations, we first annotate named entities in the documents by a named entity recognition system. We fine-tune a BERT $_{\text {LARGE }}$ (Devlin et al., 2019) model on DocRED (Yao et al., 2019), which achieves 0.91 F1 score on the DocRED validation set. Second, we link each entity mention to Wikidata by matching the mention to the name and aliases of the entities. We link the mention to the most frequent entity in Wikidata with the same name or aliases (if any). After linking the entity mentions to Wikidata, we merge the entity mentions in a document that are linked to the same entities to provide extra coreference information. Finally, each entity is associated with a set of documents that contain the entity.

Noisy Reasoning Chain Filtering. In distantly supervised annotation generation, we introduce requirements that there exists at least one reasoning chain between the labeled entity pair in the text path. To alleviate the noise in reasoning chains, we ask experts to filter out frequent noisy reasoning chains that cannot induce the target relations. Denote $h, t, b$ as head, tail and bridging entities respectively. Generally, noisy reasoning chains can be categorized into two types as follows:

Type I. The relation between $h$ and $t$ is different from the relation induced from the reasoning chain. For example, relation place of deat $h$ is different from the relation induced from $h \stackrel{\text { employer }}{\longrightarrow} b \stackrel{\text { located in }}{\longrightarrow} t$. Type I accounts for $37.8 \%$ noisy reasoning chains.

Type II. There is large uncertainty in inducing the relation from the reasoning chain. For example, relation place of birth cannot be induced from the relation $h \stackrel{\text { country of citizenship }}{\longrightarrow}$ $b \stackrel{\text { capital }}{\longrightarrow} t$. Type II accounts for $62.2 \%$ noisy reasoning chains.

Human Annotation. The annotators mainly consist of undergraduate students, and receive principled training for 4 weeks on average to fully pass the test task and regular inspections. During annotation, in addition to the relational fact, we highlight the mentions of target entities and bridging entities, and provide possible reasoning chains to assist human annotation. The salary for each relation instance is determined by the average time of annotation and local labor compensation standard. We refer readers to data description in data supplement for the user interface of our annotation platform.

Adversarial Negative Instance Generation. To alleviate the obvious correlations between relations and entity names, we employ an adversarial negative instance generation strategy. Specifically, we select entity pairs that do not have relations in Wikidata but are assigned with high confidence of positive relations by RE models.

Given a positive entity pair $(h, t)$, we generate negative entity pairs by replacing one of the entities. We first train several neural models that predicts the relation between an entity pair from entity names, including a BERT-based model, a CNN-based model, an LSTM-based model, a bilinear model, and a bag-of-words model. Specifically, the BERT-based model, CNN-based model, and LSTM-based model take the concatenation of entity names as input to predict the relation score. The bilinear model predicts the relation as follows:

$$
s_{r}=\operatorname{sigmoid}\left(\mathbf{h} \mathbf{M}_{r} \mathbf{t}+b_{r}\right),
$$

where $s_{r}$ is the score of relation $r, \mathbf{M}_{r}$ and $b_{r}$ are learnable parameters. $\mathbf{h}$ and $\mathbf{t}$ are entity name embeddings obtained from BERT as follows:

$$
\begin{aligned}
\mathbf{h} & =\operatorname{BERT}(\mathbf{h}) \\
\mathbf{t} & =\operatorname{BERT}(\mathbf{t}),
\end{aligned}
$$

where $h$ and $t$ are the name of the entity pair.

After that, for each positive entity pair $(h, t)$, we generate a negative entity pair by replacing one of the entities. We first select top 100 entities as candidates using the bilinear model due to its efficiency, then select the top entities ranked by the ensemble models as negative entities.

Data Split. In positive entity pair split, we aim to split the positive entity pairs into training, development and test set, such that there is no overlap in entity names under the same positive relations, to prevent the correlation between relations and entity names. Specifically, for each positive relation, the corresponding relational facts are represented as an undirected graph, where nodes correspond to entity pairs, and there is an edge between two nodes if the entity pairs share a common entity. Then we randomly split the connected entity pairs in the graph into training, development and test set, and ensure that relations in development and test set appeared in the training set. 


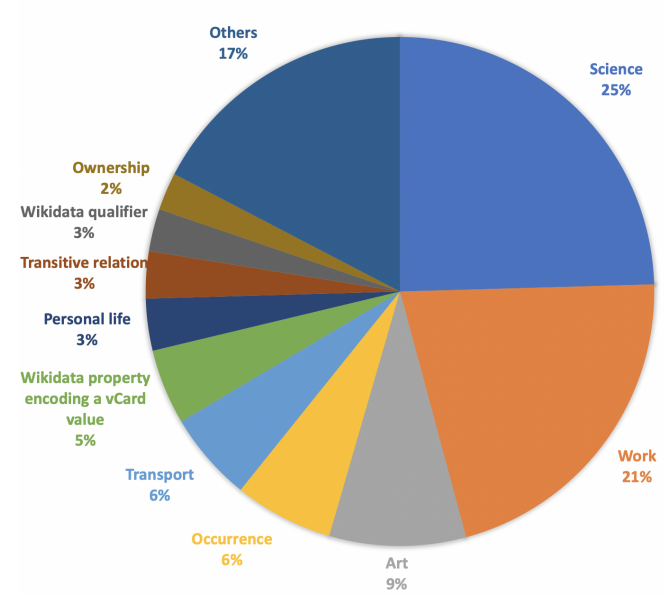

Figure 3: Relation domain distribution.

\section{B Data Distribution}

We provide distribution of relations and documents. We show relation domain distribution in Fig. 3, and the document domain distribution in Fig. 4. We can see that CodRED enjoys diversity in both relations and documents. We also compare the length of documents in CodRED and existing document-level RE datasets in Table 8. We observe that documents in CodRED are much longer than those in existing document-level RE datasets, presenting new challenges to RE systems. We refer readers to data description in data supplement for relation documentation and reasoning chain distribution.

\section{Baseline Implementation Details}

We provide implementation details of the two proposed baseline methods, including the pipeline model and the end-to-end model. For both baselines, we adopt the BERT $\mathrm{BASE}(110 \mathrm{M})$ implementation by Wolf et al. (2019).

\section{C.1 Pipeline Model.}

Intra-document Relational Graph Extraction. This phase aims to predict the relations between the entities within each document containing head or tail entities. Documents are first tokenized into word pieces (Wu et al., 2016). To extract the relation between two entities in a document, we mark the position of entity mentions. Specifically, inspired by Baldini Soares et al. (2019), we adopt special tokens as entity markers and insert them to the start and end of all mentions of an entity. Four special tokens (i.e., $\{\text { [UNUSEDi }\}_{i=0}^{3}$ from BERT vocabulary) are used to mark the start and end of two entities in a document.

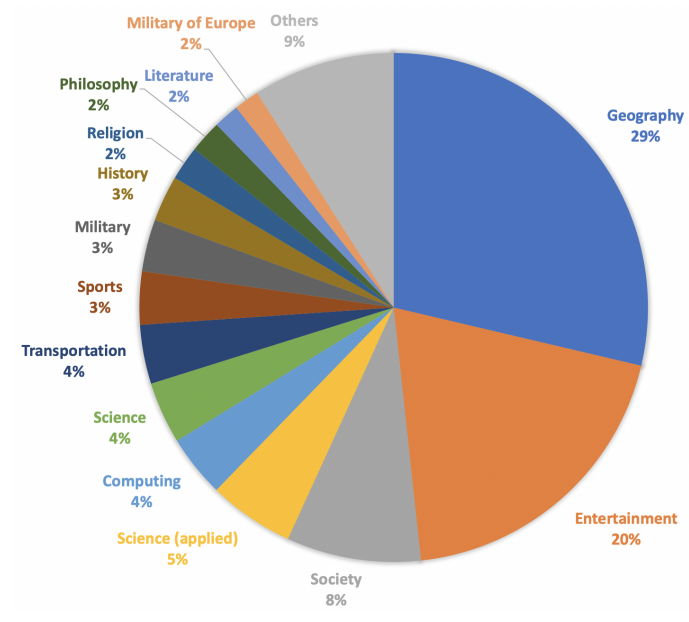

Figure 4: Document domain distribution.

\begin{tabular}{lr}
\hline Dataset & Words/Doc. \\
\hline BC5CDR (Li et al., 2016) & 118 \\
DocRED (Yao et al., 2019) & 198 \\
DialogRE (Yu et al., 2020) & 226 \\
CodRED & 2,416 \\
\hline
\end{tabular}

Table 8: Comparison of average document length between CodRED and document-level RE datasets.

After marking the entity mentions, we select relevant text in documents to encode. Since the documents in CodRED are typically very long, and the document length usually exceeds the 512 maximum input length of BERT, we extract text snippets surrounding the two entities in the document. Specifically: (1) if the distance between the nearest mentions of two entities is less than 512 , we use the 512 tokens centered on the nearest mentions; (2) otherwise we extract 255 tokens centered on the first mention of each entity, and concatenate them to obtain the input tokens. A snippet will be shifted accordingly if the span encounters the document boundaries. A [CLS] token is put at the beginning, and a [SEP] token is concatenated at the end of the input tokens. Then we feed the tokens into BERT and take the [CLS] embedding in the last layer as the entity pair representation.

Finally, the entity pair representation is fed into a fully connected layer followed by a softmax layer to obtain the relation distribution. The target relations are labeled by distant supervision as in Yao et al. (2019). The intra-document relational graph extraction model achieves $53.75 \mathrm{~F} 1$ score on the validation set of DocRED (Yao et al., 2019).

Hyperparameters. The hyperparameters are selected by grid search based on AUC metric on the 
validation set. The learning rate is $3 \mathrm{e}-5$, selected from $\{2 \mathrm{e}-5,3 \mathrm{e}-5,5 \mathrm{e}-5\}$. The batch size is 32 , selected from $\{16,32,64\}$. In cross-document relation reasoning phase, the dimension of entity type embedding and relation embedding is 256 , selected from $\{128,256\}$. We train our intra-document relational graph extraction model on 4 GeForce RTX 2080Ti GPUs for 2 epochs, which takes about 12 hours. The cross-document relation reasoning model is trained on a GeForce RTX 2080Ti GPUs for 20 epochs, which takes about 0.5 hours.

\section{C.2 End-to-end Model}

We provide details about the end-to-end model, including intra-document relation prediction and cross-document relation prediction. For intradocument relation prediction, we adopt the same approach in the pipeline model. Here we introduce details of cross-document relation prediction, including text path encoding and path aggregation.

Text Path Encoding. Given a text path $\left(d_{h}^{i}, d_{t}^{i}\right)$ of an entity pair $(h, t)$, we first encode it into representation. The text path encoding largely follows the implementation of the encoder of intradocument relational graph extraction model in the pipeline method. Documents are first concatenated and tokenized. Then entity markers are inserted to the start and end of all mentions of head, tail and bridging entities. We adopt unused tokens $\{\text { [UNUSEDi] }\}_{i=0}^{83}$ from BERT vocabulary as entity markers. $\{\text { [UNUSEDi] }\}_{i=0}^{3}$ are used to mark the start and end of head and tail entities. Bridging entities are marked by $\left\{[\text { UNUSEDi] }\}_{i=4}^{83}\right.$ according to their occurrence order in the document containing the head entity. Next we select relevant text snippets surrounding the head/tail entity in the document in a similar approach to pipeline model. Finally we feed the tokens into BERT and take the [CLS ] embedding in the last layer as the text path representation $\mathbf{p}_{i}$.

Path Aggregation. Given the representations of paths $\left\{\mathbf{p}_{i}\right\}_{i=0}^{K}$ between the entity pair, to select meaningful paths in the presence of noise, we adopt selective attention (Lin et al., 2016) to obtain the aggregated entity pair representation $\mathrm{x}$ as follows:

$$
\mathbf{x}=\sum_{i \in K} \alpha_{i} \mathbf{p}_{i}
$$

where $\alpha_{i}$ is the weight of path $p_{i}$, and is defined as:

$$
\alpha_{i}=\frac{\exp \left(e_{i}\right)}{\sum_{k \in K} \exp \left(e_{k}\right)},
$$

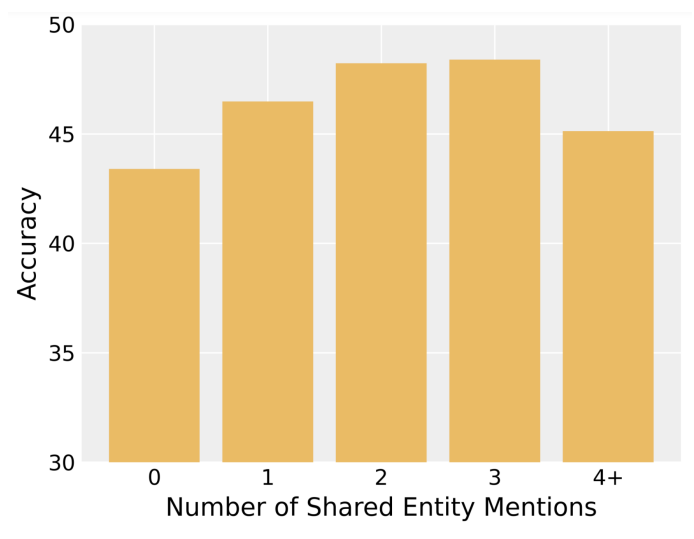

Figure 5: Accuracies of relation classification on text paths with different numbers of shared entity mentions on the development set.

where $e_{i}$ is the attention score of the path $p_{i}$, which indicates how well the path $p_{i}$ and the query relation $r$ matches. Informative paths are expected to have higher attention scores. The attention score $e_{i}$ is given by:

$$
e_{i}=\mathbf{p}_{i} \mathbf{r}
$$

where $\mathbf{r}$ is the query embedding of relation $r$.

Hyperparameters. The hyperparameters are selected by grid search according to the AUC metric on the validation set. The learning rate is $3 \mathrm{e}-5$, selected from $\{2 \mathrm{e}-5,3 \mathrm{e}-5,5 \mathrm{e}-5\}$. The batch size is selected 16 , selected from $\{8,16,32\}$. We also choose the weight decay value 0.01 . We train our model on 4 GeForce RTX 2080Ti GPUs for 10 epochs, which takes about 5 hours.

\section{Further Analysis}

Performance w.r.t. Bridging Entities. Cross-doc $\mathrm{RE}$ requires cross-document reasoning via bridging entities. To investigate the challenge of crossdocument reasoning with respect to possible bridging entity mentions, we report the model performance on text paths with different numbers of shared entity mentions between the two documents. Specifically, each text path (including positive and negative ones) is treated as an independent instance. We train a relation classification model that consists of a text path encoder and a relation predictor. Then we divide 2,558 positive text paths in the development set into subsets according to the number of shared entity mentions in the snippet, and report the accuracy of positive relation classification on each subset in Fig. 5. We observe that with the increase of shared entity mentions, the performance first improves slightly and then drops significantly. 
We hypothesize the reason is that (1) when there are few shared entity mentions, increased shared entity mention number indicates smaller semantic gaps and more alternative reasoning chains between the documents; (2) when there is a number of shared entity mentions, further increment in the number will lead to complex context and severe distractions, making the reasoning process more challenging.

\section{E Human Annotation.}

During annotation, in addition to the relational fact, we highlight the mentions of target entities and bridging entities, and provide possible reasoning chains to assist human annotation. Fig. 6 shows the user interface of our annotation platform.

\section{F Data Distribution}

We provide the list of relations and their descriptions in Wikidata in Table 9, 10, 11, 12, 13 and 14. 


\begin{tabular}{|c|c|c|}
\hline Wikidata ID & Name & Description \\
\hline P16 & highway system & system (or specific country specific road type) of which the highway is a part \\
\hline P17 & country & sovereign state of this item; don't use on humans \\
\hline P19 & place of birth & $\begin{array}{l}\text { most specific known (e.g. city instead of country, or hospital instead of city) birth location of a } \\
\text { person, animal or fictional character }\end{array}$ \\
\hline $\mathrm{P} 20$ & place of death & $\begin{array}{l}\text { most specific known (e.g. city instead of country, or hospital instead of city) death location of a } \\
\text { person, animal or fictional character }\end{array}$ \\
\hline P22 & father & male parent of the subject. For stepfather, use "stepparent" (P3448) \\
\hline $\mathrm{P} 25$ & mother & female parent of the subject. For stepmother, use "stepparent" (P3448) \\
\hline $\mathrm{P} 26$ & spouse & $\begin{array}{l}\text { the subject has the object as their spouse (husband, wife, partner, etc.). Use "unmarried partner" } \\
\text { (P451) for non-married companions }\end{array}$ \\
\hline P30 & continent & continent of which the subject is a part \\
\hline P36 & capital & primary city of a country, state or other type of administrative territorial entity \\
\hline P38 & currency & currency used by item \\
\hline P39 & position held & subject currently or formerly holds the object position or public office \\
\hline $\mathrm{P} 40$ & child & subject has the object in their family as their offspring son or daughter (independently of their age) \\
\hline P50 & author & main creator(s) of a written work (use on works, not humans) \\
\hline P53 & family & family, including dynasty and nobility houses. Not family name (use P734 for family name). \\
\hline P54 & $\begin{array}{l}\text { member of sports } \\
\text { team }\end{array}$ & sports teams or clubs that the subject currently represents or formerly represented \\
\hline P57 & director & director(s) of film, TV-series, stageplay, video game or similar \\
\hline P58 & screenwriter & person(s) who wrote the script for subject item \\
\hline P59 & constellation & $\begin{array}{l}\text { the area of the celestial sphere of which the subject is a part (from a scientific standpoint, not an } \\
\text { astrological one) }\end{array}$ \\
\hline P61 & $\begin{array}{l}\text { discoverer or inven- } \\
\text { tor }\end{array}$ & the entity who discovered, first described, invented, or developed this discovery or invention \\
\hline P69 & educated at & educational institution attended by subject \\
\hline P85 & anthem & subject's official anthem \\
\hline P86 & composer & person(s) who wrote the music [for lyricist, use "lyrics by" (P676)] \\
\hline P101 & field of work & specialization of a person or organization; see P106 for the occupation \\
\hline P102 & $\begin{array}{l}\text { member of politi- } \\
\text { cal party }\end{array}$ & the political party of which this politician is or has been a member \\
\hline P108 & employer & person or organization for which the subject works or worked \\
\hline P112 & founded by & founder or co-founder of this organization, religion or place \\
\hline P113 & airline hub & airport that serves as a hub for an airline \\
\hline P114 & airline alliance & alliance the airline belongs to \\
\hline P115 & home venue & home stadium or venue of a sports team or applicable performing arts organization \\
\hline P118 & league & league in which team or player plays or has played in \\
\hline P119 & place of burial & $\begin{array}{l}\text { location of grave, resting place, place of ash-scattering, etc, (e.g. town/city or cemetery) for a } \\
\text { person or animal. There may be several places: e.g. re-burials, cenotaphs, parts of body buried } \\
\text { separately. }\end{array}$ \\
\hline P121 & item operated & equipment, installation or service operated by the subject \\
\hline $\mathrm{P} 122$ & $\begin{array}{l}\text { basic form of gov- } \\
\text { ernment }\end{array}$ & subject's government \\
\hline P123 & publisher & organization or person responsible for publishing books, periodicals, games or software \\
\hline P126 & maintained by & $\begin{array}{l}\text { person or organization in charge of keeping the subject (for instance an infrastructure) in function- } \\
\text { ing order }\end{array}$ \\
\hline P127 & owned by & owner of the subject \\
\hline P129 & $\begin{array}{l}\text { physically interacts } \\
\text { with }\end{array}$ & physical entity that the subject interacts with \\
\hline P131 & $\begin{array}{l}\text { located in the ad- } \\
\text { ministrative territo- } \\
\text { rial entity }\end{array}$ & $\begin{array}{l}\text { the item is located on the territory of the following administrative entity. Use P276 (location) for } \\
\text { specifying the location of non-administrative places and for items about events }\end{array}$ \\
\hline P135 & movement & literary, artistic, scientific or philosophical movement associated with this person or work \\
\hline P136 & genre & $\begin{array}{l}\text { creative work's genre or an artist's field of work (P101). Use main subject (P921) to relate creative } \\
\text { works to their topic }\end{array}$ \\
\hline P137 & operator & $\begin{array}{l}\text { person or organization that operates the equipment, facility, or service; use country for diplomatic } \\
\text { missions }\end{array}$ \\
\hline P138 & named after & entity or event that inspired the subject's name, or namesake (in at least one language) \\
\hline $\mathrm{P} 140$ & religion & religion of a person, organization or religious building, or associated with this subject \\
\hline P144 & based on & the work(s) used as the basis for subject item \\
\hline P149 & architectural style & architectural style of a structure \\
\hline P150 & $\begin{array}{l}\text { contains adminis- } \\
\text { trative territorial } \\
\text { entity }\end{array}$ & (list of) direct subdivisions of an administrative territorial entity \\
\hline P155 & follows & $\begin{array}{l}\text { immediately prior item in a series of which the subject is a part [if the subject has replaced the } \\
\text { preceding item, e.g. political offices, use "replaces" (P1365)] }\end{array}$ \\
\hline
\end{tabular}

Table 9: Relation list of CodRED, including Wikidata IDs, names and descriptions of relations. 


\begin{tabular}{|c|c|c|}
\hline Wikidata ID & Name & Description \\
\hline P156 & followed by & $\begin{array}{l}\text { immediately following item in a series of which the subject is a part [if the subject has been } \\
\text { replaced, e.g. political offices, use "replaced by" (P1366)] }\end{array}$ \\
\hline P159 & $\begin{array}{l}\text { headquarters loca- } \\
\text { tion }\end{array}$ & $\begin{array}{l}\text { specific location where an organization's headquarters is or has been situated. Inverse property of } \\
\text { "occupant" (P466). }\end{array}$ \\
\hline P161 & cast member & $\begin{array}{l}\text { actor performing live for a camera or audience [use "character role" (P453) and/or "name of the } \\
\text { character role" (P4633) as qualifiers] [use "voice actor" (P725) for voice-only role] }\end{array}$ \\
\hline P162 & producer & $\begin{array}{l}\text { person(s) who produced the film, musical work, theatrical production, etc. (for film, this does not } \\
\text { include executive producers, associate producers, etc.) [for production company, use P272, video } \\
\text { games - use P178] }\end{array}$ \\
\hline P169 & $\begin{array}{l}\text { chief executive offi- } \\
\text { cer }\end{array}$ & highest-ranking corporate officer appointed as the CEO within an organization \\
\hline P170 & creator & maker of this creative work or other object (where no more specific property exists) \\
\hline $\mathrm{P} 171$ & parent taxon & closest parent taxon of the taxon in question \\
\hline P175 & performer & performer involved in the performance or the recording of a musical work \\
\hline P176 & manufacturer & manufacturer or producer of this product \\
\hline P177 & crosses & obstacle (body of water, road, ...) which this bridge crosses over or this tunnel goes under \\
\hline $\mathrm{P} 178$ & developer & organisation or person that developed the item \\
\hline P179 & series & subject is part of a series, the sum of which constitutes the object \\
\hline $\mathrm{P} 180$ & depicts & depicted entity (see also P921: main subject) \\
\hline P184 & doctoral advisor & person who supervised the doctorate or PhD thesis of the subject \\
\hline P190 & $\begin{array}{l}\text { twinned adminis- } \\
\text { trative body }\end{array}$ & $\begin{array}{l}\text { twin towns, sister cities, twinned municipalities and other localities that have a partnership or } \\
\text { cooperative agreement, either legally or informally acknowledged by their governments }\end{array}$ \\
\hline P193 & $\begin{array}{l}\text { main building con- } \\
\text { tractor }\end{array}$ & the main organization responsible for construction of this structure or building \\
\hline P197 & adjacent station & the stations next to this station, sharing the same line(s) \\
\hline P199 & business division & divisions of this organization \\
\hline P205 & basin country & country that have drainage to/from or border the body of water \\
\hline P206 & $\begin{array}{l}\text { located in or next } \\
\text { to body of water }\end{array}$ & sea, lake or river \\
\hline P241 & military branch & branch to which this military unit, award, office, or person belongs, e.g. Royal Navy \\
\hline P264 & record label & brand and trademark associated with the marketing of subject music recordings and music videos \\
\hline P272 & $\begin{array}{l}\text { production com- } \\
\text { pany }\end{array}$ & company that produced this film, audio or performing arts work \\
\hline P276 & location & $\begin{array}{l}\text { location of the item, physical object or event is within. In case of an administrative entity use } \\
\text { P131. In case of a distinct terrain feature use P706. }\end{array}$ \\
\hline P279 & subclass of & $\begin{array}{l}\text { all instances of these items are instances of those items; this item is a class (subset) of that item. } \\
\text { Not to be confused with P31 (instance of) }\end{array}$ \\
\hline $\mathrm{P} 282$ & writing system & alphabet, character set or other system of writing used by a language, supported by a typeface \\
\hline P286 & head coach & $\begin{array}{l}\text { on-field manager or head coach of a sports club (not to be confused with a general manager P505, } \\
\text { which is not a coaching position) or person }\end{array}$ \\
\hline P287 & designed by & person(s) that designed the item \\
\hline P291 & $\begin{array}{l}\text { place of publica- } \\
\text { tion }\end{array}$ & geographical place of publication of the edition (use 1st edition when referring to works) \\
\hline P306 & operating system & operating system (OS) on which a software works or the OS installed on hardware \\
\hline P355 & subsidiary & subsidiary of a company or organization, opposite of parent organization (P749) \\
\hline P360 & is a list of & common element between all listed items \\
\hline P361 & part of & $\begin{array}{l}\text { object of which the subject is a part (it's not useful to link objects which are themselves parts of } \\
\text { other objects already listed as parts of the subject). Inverse property of "has part" (P527, see also } \\
\text { "has parts of the class" (P2670)). }\end{array}$ \\
\hline P366 & use & main use of the subject (includes current and former usage) \\
\hline P375 & $\begin{array}{l}\text { space launch vehi- } \\
\text { cle }\end{array}$ & type of rocket or other vehicle for launching subject payload into outer space \\
\hline P397 & $\begin{array}{l}\text { parent astronomi- } \\
\text { cal body }\end{array}$ & major astronomical body the item belongs to \\
\hline P398 & $\begin{array}{l}\text { child astronomical } \\
\text { body }\end{array}$ & minor body that belongs to the item \\
\hline P400 & platform & $\begin{array}{l}\text { platform for which a work was developed or released, or the specific platform version of a software } \\
\text { product }\end{array}$ \\
\hline P403 & $\begin{array}{l}\text { mouth of the water- } \\
\text { course }\end{array}$ & the body of water to which the watercourse drains \\
\hline P404 & game mode & a video game's available playing mode(s) \\
\hline P408 & software engine & software engine employed by the subject item \\
\hline P411 & canonization status & stage in the process of attaining sainthood per the subject's religious organization \\
\hline P414 & stock exchange & exchange on which this company is traded \\
\hline P421 & $\begin{array}{l}\text { located in time } \\
\text { zone }\end{array}$ & time zone for this item \\
\hline $\mathrm{P} 425$ & $\begin{array}{l}\text { field of this occupa- } \\
\text { tion }\end{array}$ & $\begin{array}{l}\text { activity corresponding to this occupation (use only for occupations - for people use Property:P101, } \\
\text { for companies use P452) }\end{array}$ \\
\hline P437 & distribution & method (or type) of distribution for the subject \\
\hline P449 & original network & network(s) the radio or television show was originally aired on, including \\
\hline
\end{tabular}

Table 10: Relation list of CodRED, including Wikidata IDs, names and descriptions of relations. 


\begin{tabular}{|c|c|c|}
\hline Wikidata ID & Name & Description \\
\hline $\mathrm{P} 451$ & partner & someone in a relationship without being married. Use "spouse" for married couples. \\
\hline P452 & industry & industry of company or organization \\
\hline P460 & $\begin{array}{l}\text { said to be the same } \\
\text { as }\end{array}$ & this item is said to be the same as that item, but the statement is disputed \\
\hline P461 & opposite of & item that is the opposite of this item \\
\hline P462 & color & color of subject \\
\hline P463 & member of & $\begin{array}{l}\text { organization or club to which the subject belongs. Do not use for membership in ethnic or social } \\
\text { groups, nor for holding a position such as a member of parliament (use P39 for that). }\end{array}$ \\
\hline P479 & input method & input method or device used to interact with a software product \\
\hline P483 & recorded at & studio or location where a musical composition was recorded \\
\hline P485 & archives at & the institution holding the subject's archives \\
\hline P488 & chairperson & presiding member of an organization, group or body \\
\hline P489 & $\begin{array}{l}\text { currency symbol } \\
\text { description }\end{array}$ & item with description of currency symbol \\
\hline P495 & country of origin & country of origin of this item (creative work, food, phrase, product, etc.) \\
\hline P504 & home port & $\begin{array}{l}\text { home port of the vessel (if different from "ship registry"): For civilian ships, the primary port from } \\
\text { which the ship operates. Port of registry P532 should be listed in "Ship registry". For warships, } \\
\text { this will be the ship's assigned naval base }\end{array}$ \\
\hline P509 & cause of death & $\begin{array}{l}\text { underlying or immediate cause of death. Underlying cause (e.g. car accident, stomach cancer) } \\
\text { preferred. Use 'manner of death' (P1196) for broadest category, e.g. natural causes, accident, } \\
\text { homicide, suicide }\end{array}$ \\
\hline P511 & honorific prefix & word or expression used before a name, in addressing or referring to a person \\
\hline P512 & academic degree & academic degree that the person holds \\
\hline P516 & powerplant & equipment or engine used to power the subject \\
\hline P520 & armament & equippable weapon item for the subject \\
\hline P521 & $\begin{array}{l}\text { scheduled service } \\
\text { destination }\end{array}$ & $\begin{array}{l}\text { airport or station connected by regular direct service to the subject; for the destination of a trip see } \\
\text { P1444 }\end{array}$ \\
\hline P523 & $\begin{array}{l}\text { temporal range } \\
\text { start }\end{array}$ & the start of a process or appearance of a life form relative to the geologic time scale \\
\hline P527 & has part & part of this subject. Inverse property of "part of" (P361). See also "has parts of the class" (P2670) \\
\hline P546 & docking port & intended docking port for a spacecraft \\
\hline P551 & residence & the place where the person is or has been, resident \\
\hline P553 & website account on & $\begin{array}{l}\text { a website that the person or organization has an account on (use with P554) Note: only used with } \\
\text { reliable source or if the person or organization disclosed it. }\end{array}$ \\
\hline P559 & terminus & the feature (intersecting road, train station, etc.) at the end of a linear feature \\
\hline P598 & commander of & for persons who are notable as commanding officers, the units they commanded \\
\hline P607 & conflict & battles, wars or other military engagements in which the person or item participated \\
\hline P608 & exhibition history & exhibitions where the item is or was displayed \\
\hline P610 & highest point & point with highest elevation in a region, on a path, of a race \\
\hline P611 & religious order & order of monks or nuns to which an individual or religious house belongs \\
\hline P629 & $\begin{array}{l}\text { edition or transla- } \\
\text { tion of }\end{array}$ & is an edition or translation of this entity \\
\hline P658 & tracklist & songs contained in this item \\
\hline P664 & organizer & person or institution organizing an event \\
\hline P674 & characters & $\begin{array}{l}\text { characters which appear in this item (like plays, operas, operettas, books, comics, films, TV series, } \\
\text { video games) }\end{array}$ \\
\hline P676 & lyrics by & author of song lyrics; also use P86 for music composer \\
\hline P703 & found in taxon & the taxon in which the item can be found \\
\hline P706 & $\begin{array}{l}\text { located on terrain } \\
\text { feature }\end{array}$ & $\begin{array}{l}\text { located on the specified landform. Should not be used when the value is only politi- } \\
\text { cal/administrative (P131) or a mountain range (P4552). }\end{array}$ \\
\hline P707 & satellite bus & general model on which multiple-production satellite spacecraft is based \\
\hline P710 & participant & $\begin{array}{l}\text { person, group of people or organization (object) that actively takes/took part in an event or process } \\
\text { (subject). Preferably qualify with "object has role" (P3831). Use P1923 for team participants. }\end{array}$ \\
\hline P725 & voice actor & $\begin{array}{l}\text { performer of a spoken role in a creative work such as animation, video game, radio drama, or } \\
\text { dubbing over [use "character role" (P453) as qualifier] [use "cast member" (P161) for live acting] }\end{array}$ \\
\hline P737 & influenced by & $\begin{array}{l}\text { this person, idea, etc. is informed by that other person, idea, etc., e.g. "Heidegger was influenced } \\
\text { by Aristotle". }\end{array}$ \\
\hline P739 & ammunition & cartridge or other ammunition used by the subject weapon \\
\hline P740 & $\begin{array}{l}\text { location of forma- } \\
\text { tion }\end{array}$ & location where a group or organization was formed \\
\hline P747 & has edition & link to an edition of this item \\
\hline P749 & parent organization & parent organization of an organisation, opposite of subsidiaries (P355) \\
\hline P750 & distributor & distributor of a creative work; distributor for a record label \\
\hline P751 & introduced feature & feature introduced by this version of a product item \\
\hline
\end{tabular}

Table 11: Relation list of CodRED, including Wikidata IDs, names and descriptions of relations. 


\begin{tabular}{|c|c|c|}
\hline Wikidata ID & Name & Description \\
\hline P767 & $\begin{array}{l}\text { contributor(s) to } \\
\text { the creative work }\end{array}$ & person or organization that contributed to a subject: co-creator of a creative work \\
\hline P769 & $\begin{array}{l}\text { significant drug in- } \\
\text { teraction }\end{array}$ & $\begin{array}{l}\text { clinically significant interaction between two pharmacologically active substances (i.e., drugs } \\
\text { and/or active metabolites) where concomitant intake can lead to altered effectiveness or adverse } \\
\text { drug events. }\end{array}$ \\
\hline P790 & approved by & item is approved by other item(s) [qualifier: statement is approved by other item(s)] \\
\hline P793 & significant event & significant or notable events associated with the subject \\
\hline P800 & notable work & notable scientific, artistic or literary work, or other work of significance among subject's works \\
\hline P832 & public holiday & official public holiday that occurs in this place in its honor, usually a non-working day \\
\hline P840 & narrative location & the narrative of the work is set in this location \\
\hline P852 & ESRB rating & North American video game content rating - appropriate values are on property's talk page \\
\hline P859 & sponsor & organization or individual that sponsors this item \\
\hline P880 & CPU & central processing unit found within the subject item \\
\hline P915 & filming location & actual place where this scene/film was shot. For the setting, use "narrative location" (P840) \\
\hline P921 & main subject & primary topic of a work (see also P180: depicts) \\
\hline P924 & medical treatment & treatment that might be used to heal the medical condition \\
\hline P931 & $\begin{array}{l}\text { place served by } \\
\text { transport hub }\end{array}$ & territorial entity or entities served by this transport hub (airport, train station, etc.) \\
\hline P937 & work location & location where persons were active \\
\hline P941 & inspired by & work, human, place or event which inspired this creative work or fictional entity \\
\hline P944 & $\begin{array}{l}\text { Code of nomencla- } \\
\text { ture }\end{array}$ & the Code that governs the scientific name of this taxon \\
\hline P945 & allegiance & the country (or other power) that the person, or organization, served \\
\hline P974 & tributary & stream or river that flows into this main stem (or parent) river \\
\hline P1001 & $\begin{array}{l}\text { applies to jurisdic- } \\
\text { tion }\end{array}$ & $\begin{array}{l}\text { the item (an institution, law, public office ...) or statement belongs to or has power over or applies } \\
\text { to the value (a territorial jurisdiction: a country, state, municipality, ...) }\end{array}$ \\
\hline P1027 & conferred by & person or organization who awards a prize to or bestows an honor upon a recipient \\
\hline P1037 & director/manager & person who manages any kind of group \\
\hline P1038 & relative & $\begin{array}{l}\text { family member (qualify with "type of kinship", P1039; for direct family member please use } \\
\text { specific property) }\end{array}$ \\
\hline P1050 & medical condition & any state relevant to the health of an organism, including diseases and positive conditions \\
\hline P1056 & $\begin{array}{l}\text { product or material } \\
\text { produced }\end{array}$ & material or product produced by a government agency, business, industry, facility, or process \\
\hline P1066 & student of & person who has taught this person \\
\hline P1071 & $\begin{array}{l}\text { location of final as- } \\
\text { sembly }\end{array}$ & place where the item was made; location of final assembly \\
\hline P1072 & readable file format & file format a program can open and read \\
\hline P1073 & writable file format & file format a program can create and/or write to \\
\hline P1079 & launch contractor & organization contracted to launch the rocket \\
\hline P1080 & $\begin{array}{l}\text { from fictional uni- } \\
\text { verse }\end{array}$ & subject's fictional entity is in the object narrative. See also P1441 and P1445 \\
\hline P1142 & political ideology & political ideology of this organization or person \\
\hline P1158 & location of landing & location where the craft landed \\
\hline P1192 & connecting service & service stopping at a station \\
\hline P1303 & instrument & musical instrument that a person plays \\
\hline P1308 & officeholder & person who holds an office \\
\hline P1327 & $\begin{array}{l}\text { professional or } \\
\text { sports partner }\end{array}$ & person a professional or athlete works with \\
\hline P1336 & $\begin{array}{l}\text { territory claimed } \\
\text { by }\end{array}$ & administrative divisions that claim control of a given area \\
\hline P1343 & $\begin{array}{l}\text { described by } \\
\text { source }\end{array}$ & dictionary, encyclopaedia, etc. where this item is described \\
\hline P1344 & participant of & event a person or an organization was/is a participant in, inverse of P710 or P1923 \\
\hline P1346 & winner & winner of an event or award - do not use for wars or battles \\
\hline P1365 & replaces & $\begin{array}{l}\text { person or item replaced. Use P1398 (structure replaces) for structures. Use P155 (follows) if the } \\
\text { previous item was not replaced or if predecessor and successor are identical. }\end{array}$ \\
\hline P1366 & replaced by & $\begin{array}{l}\text { other person or item which continues the item by replacing it in its role. Use P156 (followed by) } \\
\text { if the item is not replaced (e.g. books in a series), nor identical, but adds to the series without } \\
\text { dropping the role of this item in that series }\end{array}$ \\
\hline P1387 & political alignment & political position within the political spectrum \\
\hline P1389 & $\begin{array}{l}\text { product certifica- } \\
\text { tion }\end{array}$ & certification for a product, qualify with P1001 ("applies to jurisdiction") if needed \\
\hline P1399 & convicted of & crime a person was convicted of \\
\hline P1408 & $\begin{array}{l}\text { licensed to broad- } \\
\text { cast to }\end{array}$ & place that a radio/TV station is licensed/required to broadcast to \\
\hline
\end{tabular}

Table 12: Relation list of CodRED, including Wikidata IDs, names and descriptions of relations. 


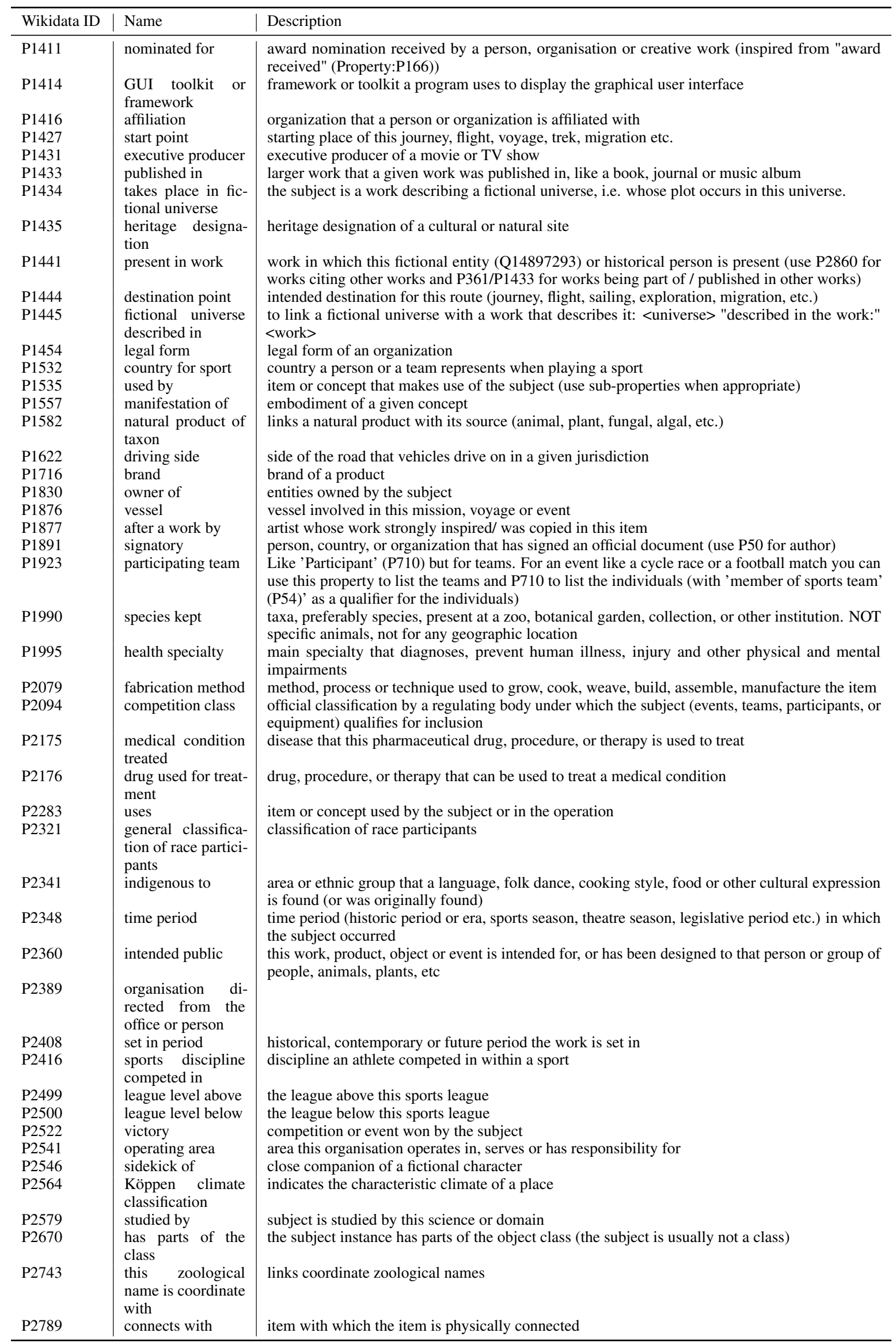

Table 13: Relation list of CodRED, including Wikidata IDs, names and descriptions of relations. 


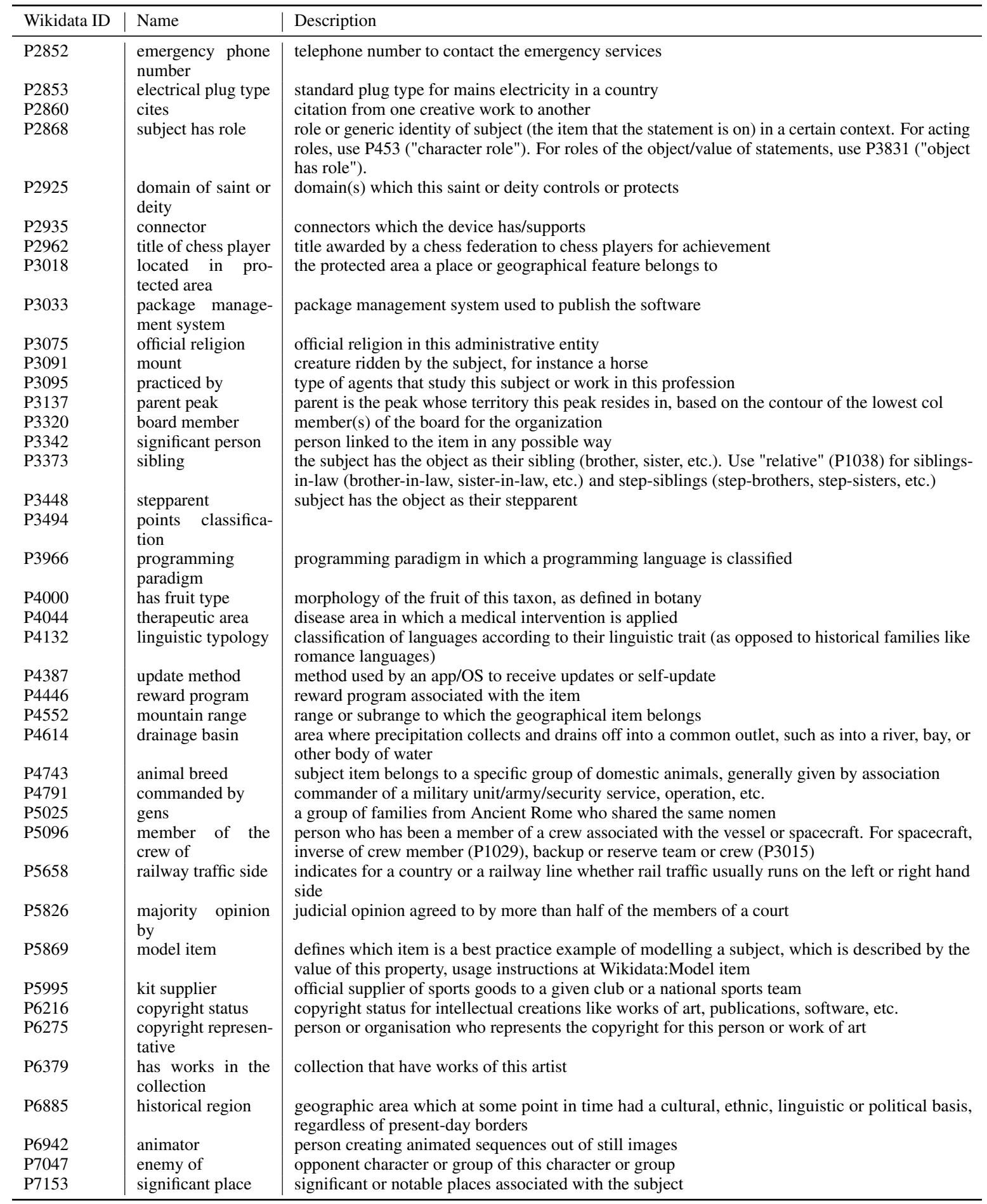

Table 14: Relation list of CodRED, including Wikidata IDs, names and descriptions of relations. 


\begin{tabular}{|c|c|c|c|}
\hline Head entity & Relation & Tail entity & Supported \\
\hline Mega-Gem & country & the United States & Yes \\
\hline
\end{tabular}

Mega-Gem is an outdoor sculpture by American artist John Fran... Delete Her work is represented in the collections of the Musée d'Orsay i... Delete

\begin{tabular}{|c|c|c|c|c|c|}
\hline \multicolumn{3}{|c|}{ Possible reasoning chains in article 1} & \multicolumn{3}{|c|}{ Possible reasoning chains in article 2} \\
\hline Head entity & Relation & Tail entity & Head entity & Relation & Tail entity \\
\hline Mega-Gem & collection & Indianapolis Museum of Art & Indianapolis Museum of Art & country & the United States \\
\hline Mega-Gem & located in the administrative territorial entity & Indianapolis & Indianapolis & country & the United States \\
\hline \multicolumn{6}{|c|}{ Head/Tail Entity in possible reasoning chains Common entities Evidence sentences } \\
\hline \multicolumn{6}{|c|}{ Article1--Mega-Gem } \\
\hline \multicolumn{6}{|c|}{ 1. Mega-Gem is an outdoor sculpture by American artist John Francis Torreano (born 1941). } \\
\hline \multirow{3}{*}{\multicolumn{6}{|c|}{ 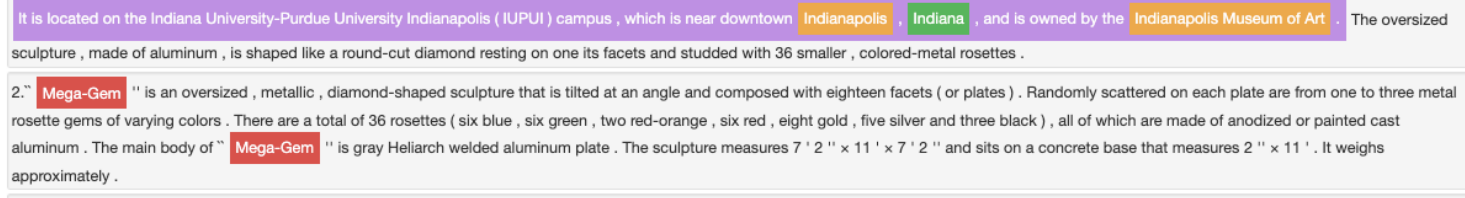 }} \\
\hline & & & & & \\
\hline & & & & & \\
\hline \multicolumn{6}{|c|}{$\begin{array}{l}\text { 3." Mega-Gem " was fabricated in } 1989 \text { with the resources of Cincinnati art dealer Carl Solway. It was presented at the Chicago International Art Exposition, where it was located on the Navy Pier in } \\
\text { Chicago , Illinois . The presentation of "Mega-Gem " was promoted through posters and buttons proclaiming the sculpture to be the largest diamond in the world, weighing over } 360 \text { million carats ." } \\
\text { Mega-Gem " was considered by Torreano to be one of a series of " oxy-gem " sculptures, playing on the oxymoron of combining precious gems with materials of lesser value , such as a " plywood gem, " " } \\
\text { gold gem, " and " Mega-Gem " as " aluminum gem. " " Mega-Gem "is one of Torreano 's oversized and exaggerated jewel sculptures. }\end{array}$} \\
\hline \multicolumn{6}{|c|}{ 4.In 1989 " Mega-Gem " was presented at the Chicago International Art Exposition where it was displayed on Navy Pier along Lake Michigan in Chicago , llinois , until 1994.} \\
\hline \multicolumn{6}{|c|}{$\begin{array}{l}\text { 5.In October } 1994 \text { " Mega-Gem " was loaned to the Indianapolis Museum of Art for two years. It arrived on October } 10,1994 \text {, and was put on display in the southwest corner of Krannert Plaza, which is a } \\
\text { section of the IMA 's grounds and gardens located on the west side of the property overlooking the White River. In } 1997 \text {, after the loan period had expired, the Contemporary Art Society raised funds for" } \\
\text { Mega-Gem "to be acquired by the IMA. It remained on view in Krannert Plaza until } 2001 \text {, when it was relocated to the southeast corner of the IMA property near the intersection of } 38 \text { th Street and Michigan } \\
\text { Road. }\end{array}$} \\
\hline \multicolumn{6}{|c|}{$\begin{array}{l}\text { 6.In late January } 2009 \text { " Mega-Gem "was relocated to the IUPUI campus to make way for the creation of the IMA 's Virginia B. Fairbanks Art \& Nature Park, which opened in June } 2010 \text {." Mega-Gem " was } \\
\text { one of four IMA sculptures that were loaned to IUPUI. The others were "East Gate/West Gate " by Sasson Soffer, " Portrait of History " by Shan Zou Zhou, and " Spaces with Iron " by Will Horwitt. These four } \\
\text { IMA pieces on the IUPUI campus are part of the , which" "connects neighborhoods, entertainment facilities and the city 's five cultural districts " and includes Indiana Avenue , Massachusetts Avenue, } \\
\text { Indianapolis , Fountain Square, Indianapolis , the Wholesale District, Indlanapolis , and White River State Park. The Cultural Trail, completed in } 2013 \text { as a bike and pedestrian path, will connect Broad } \\
\text { Ripple Village, Indianapolis to downtown Indianapolis via the Monon Trail. }\end{array}$} \\
\hline \multicolumn{6}{|c|}{ 7." Mega-Gem " is situated in the courtyard north of New York Street on the IUPUI campus, east of Lecture Hall and south of Joseph T. Taylor Hall ( formerly University College ), at 815 W. Michigan Street . } \\
\hline \multicolumn{6}{|c|}{$\begin{array}{l}\text { 8." Mega-Gem " was loaned to the Indianapolis Museum of Art (IMA) by the Carl Solway Gallery from } 1994 \text { to } 1996 \text {. In } 1997 \text { the IMA Contemporary Art Society (CAS) undertook the effort to purchase the } \\
\text { sculpture and acquire it for the IMA.CAS President Dee Garrett led the fund drive for" Mega-Gem "and worked with the IMA to sell miniature gem sculptures created by Torreano in order to raise money. } \\
\text { The CAS donated" Mega-Gem " to the IMA at a gala in } 1997 \text { with John Torreano in attendance. }\end{array}$} \\
\hline \multicolumn{6}{|c|}{$\begin{array}{l}\text { 9." Mega-Gem " was acquired by the IMA in } 1997 \text { with the accession number of } 1997.6 \text {. It is credited as the Gift of Robert Shiffler, Contemporary Art Society Fund and Henry F. and Katherine D. DeBoest } \\
\text { Memorial Fund. The value of " Mega-Gem "is unknown ; however , prices for Torreano 's work have ranged from } \$ 4,000 \text { for smaller paintings to } \$ 30,000 \text { for larger pieces. }\end{array}$} \\
\hline \multicolumn{6}{|c|}{$\begin{array}{l}\text { 10.The fading paint on the rosettes has been a cause for concern in the past. In } 1996 \text {, in preparation for "Mega-Gem " 's acquisition into the Indianapolis Museum of Art collection, the rosettes were } \\
\text { returned to the artist for repainting. }\end{array}$} \\
\hline \multicolumn{6}{|c|}{ Article2--Janet Scudder } \\
\hline \multicolumn{6}{|c|}{$\begin{array}{l}\text { 12.Janet Scudder ( October } 27,1869-\text { June } 9,1940 \text { ), born Netta Deweze Frazee Scudder, was an American sculptor and painter from Terre Haute, Indiana , who is best known for her memorial } \\
\text { sculptures , bas-relief portraiture, and portrait medallions, as well as her garden sculptures and fountains. Her first major commission was the design for the seal of the New York Bar Association around } 1896 \text {. } \\
\text { Scudder ' } \mathrm{s} \text { "Frog Fountain " (1901) led to the series of sculptures and fountains for which she is best known. Later commissions included a Congressional Gold Medal honoring Domicio da Gama (Brazil 's } \\
\text { ambassador to the United States ) and a commemorative medal for Indiana 's centennial in } 1916 \text {. Scudder also displayed her work at numerous national and international exhibitions in the United States } \\
\text { and in Europe from the late } 1890 \text { s to the late } 1930 \text { s. Scudder 's autobiography, "Modeling My Life ", was published in } 1925 \text {. }\end{array}$} \\
\hline \multicolumn{6}{|c|}{$\begin{array}{l}\text { 13.Scudder received art training at the Art Academy of Cincinnati in 1887-89 and 1890-91 and the Art Institute of Chicago in 1891-92. In addition, she worked as an assistant to Lorado Taft during preparations } \\
\text { for the World 's Columbian Exposition in Chicago , in 1892-93, and with Frederick W. MacMonnies in Paris, France in 1894-96, while continuing her art studies at the Académie Vitti and the Académie } \\
\text { Colarossi. Scudder was a member of New York State Woman Suffrage Association, the art committee of the National American Woman Suffrage Association, and in } 1920 \text {, was elected an associate of the } \\
\text { National Academy of Design . Scudder was named a Chevalier of the French Legion of Honor in } 1925 \text { for her relief work as a Red Cross volunteer in France during World War I. }\end{array}$} \\
\hline \multicolumn{6}{|c|}{$\begin{array}{l}\text { 14.Scudder was the recipient of several awards and prizes for her artwork, including a Bronze Medal , World 's Columbian Exposition , } 1893 \text {; a Bronze Medal , Louisiana Purchase Exposition , } 1904 \text {; a Silver } \\
\text { Medal , Panama-Pacific International Exposition , } 1915 \text {; and a Silver Medal , International Exposition , } 1937 \text {, among others. }\end{array}$} \\
\hline \multicolumn{6}{|c|}{$\begin{array}{l}\text { Her work is represented in the collections of the Musée d'Orsay in the Musée d'Art Moderne de la Ville de Paris in France, and in the United States at the Library of Congress, the Metropolitan Museum of } \\
\text { Art, the Art Institute of Chicago, the Peabody Institute, Brookgreen Gardens, the Huntington Library. Art Gallery and Botanical Gardens, the Indianapolis Museum of Art ; the Indlana State Museum, the } \\
\text { Indiana Historical Society, the Swope Art Museum, and the Richmond Art Museum. }\end{array}$} \\
\hline
\end{tabular}

Figure 6: The annotation platform. Annotators are provided with relational fact and text paths. We also highlight the mentions of target entities and bridging entities, and provide possible reasoning chains to assist annotation. 